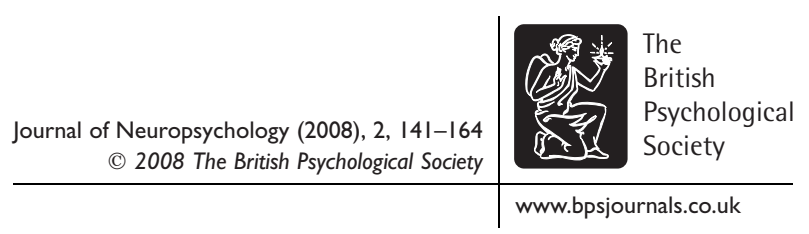

\title{
Implicit familiarity processing in congenital prosopagnosia
}

\author{
Galia Avidan ${ }^{*}$ and Marlene Behrmann ${ }^{2}$ \\ 'Department of Psychology and the Zlotowski Center for Neuroscience, \\ Ben-Gurion University of the Negev, Beer Sheva, Israel \\ ${ }^{2}$ Department of Psychology and Center for the Neural Basis of Cognition, \\ Carnegie Mellon University, Pittsburgh, PA, USA
}

\begin{abstract}
A particularly interesting and somewhat puzzling finding in the face-processing literature is that, despite the absence of overt recognition of most faces, many patients with acquired prosopagnosia (AP) exhibit evidence of intact covert face recognition of the very same faces. This phenomenon has important implications for the understanding of the mechanism underlying AP and, by extension, the mechanism underlying normal face processing. Here, we set out to examine whether individuals with congenital prosopagnosia (CP) exhibit a similar dissociation between overt and covert face recognition. We first confirmed that all six of our $\mathrm{CP}$ individuals were significantly impaired in face recognition in comparison with controls. Participants then completed a matching task with both famous and unknown faces in which they decided whether two consecutive images have the same identity or not. Critically, the level of face familiarity was orthogonal to the task at hand and this enabled us to examine whether the familiarity of a face enhanced identity matching, a finding which would implicate implicit face processing. As expected, the CP individuals were slower and less accurate than the control participants. More importantly, like the controls, the CP individuals were faster and more accurate at matching famous compared with unknown faces. Also, for both groups, matching performance on unrecognized famous faces fell at an intermediate level between performance on explicitly recognized famous faces and faces which are unknown. These results provide the first solid evidence for the existence of implicit familiarity processing in $\mathrm{CP}$ and suggest that, despite the marked impairment in explicit face recognition, these individuals still have some familiarity representation which manifests in the form of covert recognition. We discuss possible models to account for the apparent dissociation of overt and covert face processing in CP.
\end{abstract}

Prosopagnosia refers to the inability to recognize faces in individuals who have normal sensory vision and normal intelligence. The term is typically used with respect to individuals who were premorbidly normal but subsequently fail to

\footnotetext{
* Correspondence should be addressed to Dr Galia Avidan, Department of Psychology, Ben-Gurion University of the Negev, Beer Sheva 84105, Israel (e-mail: galiaa@bgu.ac.il).
} 
recognize faces following acquired brain damage. In these individuals with acquired prosopagnosia (AP), the lesion is typically to the ventral visual cortex and is sustained during adulthood (for reviews, see Barton, 2003; Bouvier \& Engel, 2006; Damasio, Damasio, \& Van Hoesen, 1982). The study of AP has a rather long history (Bodamer, 1947) and this disorder has provided a unique window into the psychological and neural substrate of face processing. In recent years, an analogous impairment in face processing to AP has been described and termed congenital prosopagnosia (CP) to reflect the fact that it is apparently lifelong in duration, arises in the absence of brain damage of any form, and occurs in individuals who have had adequate opportunity to acquire normal face-recognition skills (for recent review, see Behrmann \& Avidan, 2005). This impairment oftentimes also has a familial or a genetic basis (Grueter et al., 2007). This condition is to be differentiated from developmental prosopagnosia (DP) which, although evident from early life too as in the case of CP, is associated with brain injury incurred as a result of, for example, respiratory arrest or a major fall early in the course of development (for example, see Barton, Cherkasova, Press, Intriligator, \& O'Connor, 2003). However, it is important to note that some researchers use the term DP rather than $\mathrm{CP}$, even in cases where no apparent brain injury has occurred (e.g. Duchaine, Germine, \& Nakayama, 2007), to indicate that we cannot be certain when exactly face processing diverged from normal in these individuals. Moreover, several of the case studies described in the literature as DP would probably qualify as CP based on the definition we use here (e.g. (De Haan \& Campbell, 1991; Jones \& Tranel, 2001)). Although we cannot definitively be sure in our own cases that the disorder was present from birth, these individuals apparently have not sustained any neurological insult and so we adopt the term CP here to be clear about the absence of any obvious neurological insult.

\section{Congenital prosopagnosia: Behavioural and neural profile}

Although CP has attracted much scientific attention recently, many aspects of its behavioural profile and underlying neural mechanism are still unclear. Thus, while most researchers would agree that the hallmark of the disorder is the inability to recognize familiar faces, the extent of the impairment in other tasks related to face and non-face processing is not yet fully agreed upon (Behrmann, Avidan, Marotta, \& Kimchi, 2005; Bentin, Degutis, D'Esposito, \& Robertson, 2007; Duchaine et al., 2007; Duchaine \& Nakayama, 2004; Le Grand et al., 2006). The evidence is also somewhat mixed at the neural level. While some studies have documented robust and apparently normal facerelated activation in occipitotemporal cortex in CP (Avidan, Hasson, Malach, \& Behrmann, 2005; Hasson, Avidan, Deouell, Bentin, \& Malach, 2003) and particularly in the fusiform gyrus, the pre-eminent face-processing cortical region (Kanwisher, McDermott, \& Chun, 1997), other studies have been unable to uncover selective facerelated BOLD activation (Bentin et al., 2007; Hadjikhani \& De Gelder, 2002). Mixed findings have also been reported in the patterning of the face-selective N170 EEG and M170 MEG waveforms (Bentin, Deouell, \& Soroker, 1999; Harris, Duchaine, \& Nakayama, 2005; Kress \& Daum, 2003; Minnebusch, Suchan, Ramon, \& Daum, 2007), with some CP individuals evincing atypical waveforms but others exhibiting waveforms that closely mirror those of normal observers. In addition, some studies have begun to examine possible structural alterations in the brains of individuals with CP. Two fairly recent studies have documented reduced cortical volume in $\mathrm{CP}$, either in the temporal 
lobe (Bentin et al., 1999) or, more specifically, in the anterior portion of the temporal lobe (Behrmann, Avidan, Gao, \& Black, 2007), and both have suggested that this volumetric reduction may, at least, partially account for the impairment in face processing in CP. Consistent with this, using diffusion tensor imaging, Thomas, Avidan, Jung, \& Behrmann (2006) have revealed reduced white matter connectivity in the white matters tracts that connect the fusiform gyrus to anterior temporal and frontal regions in six individuals with CP. These findings provide the first evidence for impaired connectivity between occipitotemporal cortex and more anterior cortical regions in $\mathrm{CP}$ and the disrupted propagation of information from more posterior occipitotemporal cortex to more anterior regions may account for the behavioural impairment in $\mathrm{CP}$.

\section{Implicit face processing in prosopagnosia}

Notwithstanding the growing interest in investigating CP, a number of outstanding questions remain. Among these is whether and to what extent these individuals show evidence of implicit processing of face identity or familiarity. The answer to this question has major implications for our understanding of $\mathrm{CP}$ and its underlying mechanisms. The study of implicit processing in prosopagnosia has a relatively long and rich history, and many studies have demonstrated that AP patients, who cannot recognize famous faces explicitly, still show some implicit processing of the face identity. The evidence to support the presence of implicit processing comes from various measures including skin conductance response (SCR) (Tranel \& Damasio, 1985), event-related potentials (Bobes et al., 2003; Renault, Signoret, Debruille, Breton, \& Bolgert, 1989), eye-movement patterns (Rizzo, Hurtig, \& Damasio, 1987), and behavioural paradigms. While we do not provide an exhaustive description of these various behavioural tasks here and the reader is referred to the original papers for further details, it is important to note that there is some heterogeneity in the results of these studies and one possible explanation for the different outcomes is the use of substantially different tasks. A major distinction between the tasks is the extent to which they are direct versus indirect (Barton, Cherkasova, \& Hefter, 2004). In direct tasks, participants make identityrelated decisions about faces, even those not explicitly recognized (e.g. sorting famous faces by occupation); In contrast, in indirect tasks, the effect of facial identity or familiarity on an orthogonal task is measured. For example, participants may classify names by fame or occupation while a congruent or incongruent task-irrelevant face precedes or accompanies the name (e.g. Young, Hellawell, \& De Haan, 1988). Implicit processing is revealed by facilitated categorization of the names for congruent over incongruent name-face pairs (for recent reviews of the findings in AP and comparison of findings across different tasks see Barton et al., 2004; Bruyer, 1991; Farah, O'Reilly, \& Vecera, 1993; Schweinberger \& Burton, 2003).

In the current investigation, we employ an identity-matching task with familiar and unknown faces. This task is similar to that used by De Haan, Young, \& Newcombe (1987) in a patient with AP. This task can be categorized as 'indirect' in that it measures the facilitation of identity matching for familiar over unknown faces in a paradigm in which familiarity is orthogonal and irrelevant for the task being performed. However, it resembles direct tasks too in that the participants make judgments about the face itself rather than about some other dimension such as the name or the occupation of the individual, as described above. 


\section{Models of implicit face processing in prosopagnosia}

Whether the task taps identity or other dimensions of the face in a direct or indirect manner may engage the underlying neural mechanism differentially and several major theoretical models have been proposed to explain the possible differences. We review these models briefly and discuss them in more detail and in relation to $\mathrm{CP}$ in the Discussion section. An early model proposed by Bauer (1984) posits that there are two separate systems for face recognition such that overt recognition is mediated by the ventral visual pathway which is damaged in AP, while covert recognition by a dorsal visual limbic route which has to do more with the affective response elicited by the face and this system is preserved in AP.

An alternative model suggests that the dissociation between overt and covert processing arises from a disconnection between nodes of a circuit but exactly where this disconnection arises differs according to different theoretical proposals. In one disconnection account offered by De Haan, Bauer, \& Greve (1992), covert recognition in the absence of overt recognition in AP results from a disconnection between an intact face-processing system supporting covert recognition and a higher cognitive system enabling conscious awareness. An alternative disconnection model proposed by Burton Young, Bruce, Johnston, \& Ellis (1991) and implemented by a computer simulation instantiates a version of the classical Bruce and Young face-recognition model (Bruce \& Young, 1986). Here, the dissociation between overt and covert recognition results from a disconnection within the face-processing system itself: specifically, the connection weights between the intact face representations (face-recognition units, FRUs in the terminology of Bruce and Young model) and higher order, modality-independent representation supporting the intact final stages of recognition (person identity nodes, PINs) are decreased and cannot reach threshold to support overt recognition. Subthreshold activation, however, can result in covert recognition.

A major assumption of the disconnection models is that the face-recognition system per se is intact in AP. This assumption, however, has been challenged by empirical evidence showing that many AP patients do exhibit perceptual difficulties with faces in addition to their difficulties in recognition (Farah, 1992; Farah et al., 1993). To account for this, Farah et al. have proposed an alternative view in which overt and covert recognition are accomplished by a single neural system. On this account, and contra Burton and colleagues, face representation in AP is not intact and the crucial difference between overt and covert recognition lies in their differential sensitivity to the residual face information. Critically, the claim is that degraded residual face representations may suffice for successful performance on covert recognition tasks whereas more precise, higher quality information is required to achieve normal overt recognition.

\section{Implicit processing in CP}

A further assumption of the models outlined above is that, for implicit processing to occur, normal face representations must have been acquired premorbidly, prior to the onset of the disorder, and hence can potentially be activated. This assumption is clear in the Farah and colleagues neural network model (Farah, 1992; Farah et al., 1993) in which the network was initially fully trained to develop intact representations and was then damaged to simulate AP, but the claim that individuals must have previously acquired FRUs is also explicit in the Burton et al. account (see Schweinberger \& Burton, 2003). If the existence of pre-existing normal representations is a prerequisite 
for face processing (overt as well as covert in the context of the Farah model), then one might not expect to observe implicit processing in individuals with $\mathrm{CP}$ for whom, by definition, face representation was never normally acquired.

To date, only a few studies have examined implicit face processing in $\mathrm{CP}$, and the evidence itself is not clear-cut. Using behavioural measures, Bentin et al., (1999) found no implicit processing in their first $\mathrm{CP}$ patient and only weak, indirect evidence for implicit processing in their second CP patient (Bentin et al., 2007). Similarly, De Haan and Campbell (1991) found no clear evidence for implicit processing in a single developmental prosopagnosic participant (whom we would label $\mathrm{CP}$ given the absence of a neurological history). However, in contrast, Jones and Tranel (2001) reported an increased amplitude of skin conductance responses (SCR) for familiar compared with unknown faces which was similar to that of controls, in a child with developmental prosopagnosia (but who also fits our definition of $\mathrm{CP}$ ) with no neurological or psychiatric background.

Given these contradictory findings, we examined implicit face processing in a relatively large group of six individuals with $\mathrm{CP}$. We first assessed the explicit recognition of famous faces in each of these individuals and confirmed that it was significantly poorer than that of a group of control participants (Figure 1). We then employed a face identity matching task with famous and unknown faces in which participants made an explicit judgment about a face i.e. decided whether two consecutive images belong to the same

(a) Recognition of famous faces
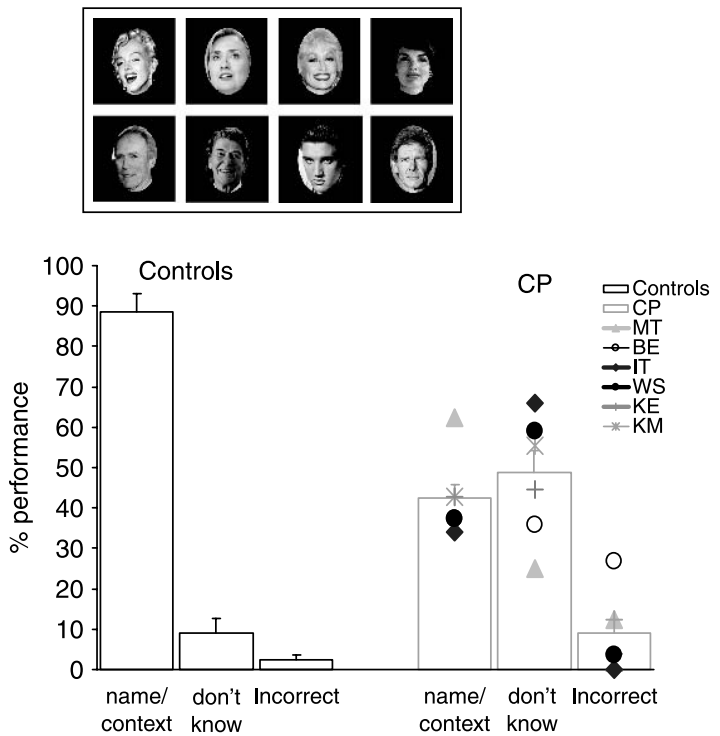

(b) Unfamiliar face discrimination
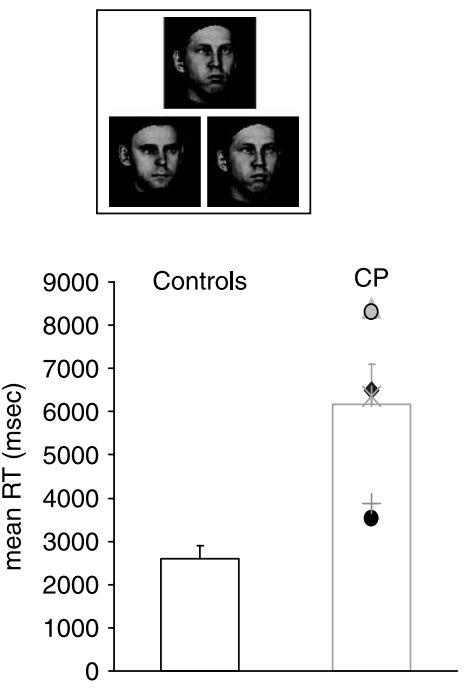

Figure I. Performance of $\mathrm{CP}$ and controls on diagnostic face-processing tests. (a) Examples of stimuli included in the famous face questionnaire; the graph shows the mean performance of the control group $(N=12$, black bars), the CP group (grey bars), and each individual symbol indicates the performance of one $\mathrm{CP}$ individual. As can be seen, all CP perform well below the normal range on this task. Error bars in all figures indicate \pm standard error of the mean across participants. (b) Examples of stimuli included in the unfamiliar face discrimination test. The graph exhibits the mean reaction time of control (black) and CP (grey) participant, as well as the individual performance of each CP individual. As can be seen reaction time for all CP participants was substantially higher than the mean RT for controls. 
person or not. A paradigm similar to this, in which the level of face familiarity is orthogonal and irrelevant to the task, has been used previously to uncover better performance for famous compared with unknown faces in normal individuals (Young, McWeeny, Hay, \& Ellis, 1986) and in an individual with AP (De Haan et al., 1987).

\section{Methods}

\section{Participants}

All participants had normal or corrected-to-normal vision, were right-handed, and consented to participate in the experiments. The protocol was approved by the Institutional Review Boards of Carnegie Mellon University and the University of Pittsburgh.

\section{Controls}

Twelve healthy individuals (nine females), aged 21-70 (mean $\pm S D 32 \pm 18$ ) participated in one fMRI scanning session during which they completed the experimental face identity matching task. This group, along with an additional group of 18 controls (total of 30 controls), completed the famous face questionnaire (see below). This large group of controls included at least two age- and gender-matched controls for each CP participant. As will be evident, we compare the performance of the $\mathrm{CP}$ individuals to both groups of control participants to evaluate the differences between the CP and normal individuals as closely as possible. Since we tested face-recognition abilities using a set of faces who are famous in the American culture (see below), we only included control participants who reported living in the US all or most of their life.

\section{$C P$}

Six healthy individuals diagnosed with CP (one male), aged between 29 and 73, participated in this study. Further details about the CP participants are provided in Table 1. None of the CP individuals had any discernible cortical lesion on conventional MRI scanning, and none had a history of any neurological or psychiatric disease. Three of these individuals (KM, MT, and BE) have participated in our previous studies and, in addition to the data reported here, more detailed behavioural profiles can be found in other related papers (Avidan et al., 2005; Behrmann et al., 2008; Behrmann et al., 2005; Humphreys, Avidan, \& Behrmann, 2007). The other three participants were systematically evaluated using a battery of face-processing tests (see also (Avidan, Thomas, \& Behrmann, 2007; Behrmann et al., 2005)) and their performance on a subset of these tasks is provided below.

Table I. Demographic information of CP participants

\begin{tabular}{lcr}
\hline CP initials & Sex & Age \\
\hline KM & F & 60 \\
MT & M & 46 \\
BE & F & 29 \\
IT & F & 73 \\
KE & F & 67 \\
WS & F & 64 \\
\hline
\end{tabular}




\section{Diagnostic tasks for CP}

As part of the diagnostic procedure, all participants were tested on a famous face questionnaire and on a face discrimination task of unfamiliar faces.

Famous face questionnaire

The questionnaire included photographs of faces of 56 celebrities, randomly intermixed with 56 photos of the faces of unknown individuals (celebrities who were famous in other countries and were verified to be unknown to a large group of US participants in pilot studies). Most pictures were taken from the internet and some were scanned from magazines. Images were cropped with a standard black oval to remove non-facial cues. Roughly half of the faces were male and the number of Caucasian and non-Caucasian faces was equated across the famous and unknown sets. The pictures were shown on a piece of paper or on a computer screen for an unlimited duration and the entire questionnaire was completed in a self-paced fashion. A response giving either the name of the individual (e.g. Bill Clinton) or any contextual information (e.g. former president of the US) was scored as correct. Other possible responses were an incorrect name (e.g. Harrison Ford in response to the picture of Bill Clinton), or 'don't know'. The images included in the questionnaire were drawn from the large collection of images used in the face identity experiment described below and were identical across all participants. The stimulus set was first tested and refined in a pilot behavioural experiment with a different set of participants to ensure that the famous faces were indeed highly recognizable and familiar. This pilot study showed a high recognition level of the famous faces $(\sim 85 \%)$ and a negligible level of recognition of the unknown faces. Critically, all CP individuals were well below the control mean on this task (see Figure 1 and the Results section for more details).

\section{Unfamiliar face discrimination}

All CP participants were tested on a simultaneous face discrimination task and their performance was compared to that of 12 normal controls who completed this task previously (Behrmann et al., 2005). In each trial, three unfamiliar faces, shown from a frontal view, appeared on the screen in a pyramid format (one face at the top and the two choice faces presented below it to the left and right) for unlimited exposure duration. Participants had to decide whether the 'target' face presented at the top matched the face on the bottom left or the bottom right (for more details about the experimental design see Behrmann et al., 2005). Accuracy was high, likely as a result of the unlimited exposure duration, and so we concentrate on reaction time (RT). Overall, CP participants were significantly slower than controls on this task $(p<.0002$; mean RT \pm standard error of the mean: controls: $2,592 \pm 319 \mathrm{msec}$; CP: 6,162 $\pm 934 \mathrm{msec}$ ). Importantly, all CP participants fell below the $95 \%$ confidence interval of control performance $(3,190 \mathrm{msec})$ and four out of the six CP participants were also significantly slower than controls when the Crawford's modified $t$ test for single case method was applied $(p<.005$; Crawford \& Garthwaite, 2002). Thus, all CP participants exhibit difficulty in matching unfamiliar faces in addition to their difficulty in face recognition (see Figure 1 and text below), further confirming the diagnosis of prosopagnosia for each individual. 


\section{Experimental tasks}

Face identity matching experiment

Stimuli included a total of 560 different pictures of faces of 112 different individuals (about 50\% female). Pictures were high-quality monochrome or colour, contained minimal, if any, obvious diagnostic cues such as hats or facial hair and were mostly downloaded from the internet. Amongst this large set of images were the photos of the 56 famous and 56 non-famous individuals from the famous faces questionnaire, as described above.

In this task, in a single trial, two face images were shown consecutively and participants decided whether the faces were of the same individual or not and indicated their response by button press (same/different). The experiment had a $3 \times 2$ design in which three different trial types were included (Figure 2): same picture, same identity (top row); different picture, different identity (middle row); and different picture, same identity (bottom row). In half of the trials, the pictures were of famous faces and, in the other half, they were of unknown faces; famous and unknown faces were always presented in separate trials such that the two faces within a pair were either from the famous or from the unknown face sets. In order that the exact same image of a face is repeated only during the 'same picture, same identity' condition, five different exemplars of each individual face were used in the experiment.

(a) Trial type

I. Same picture
Same identity

II. Different picture Different identity

III. Different picture Same identity

(b)
Famous

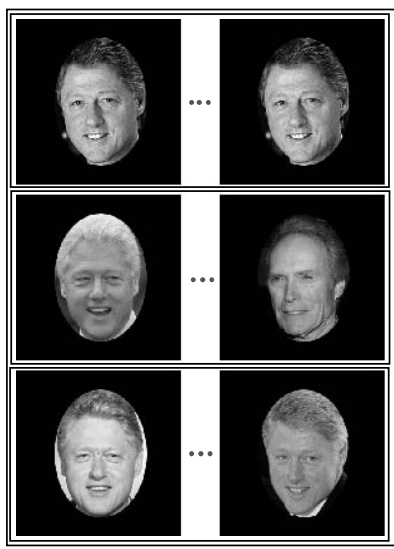

Unknown

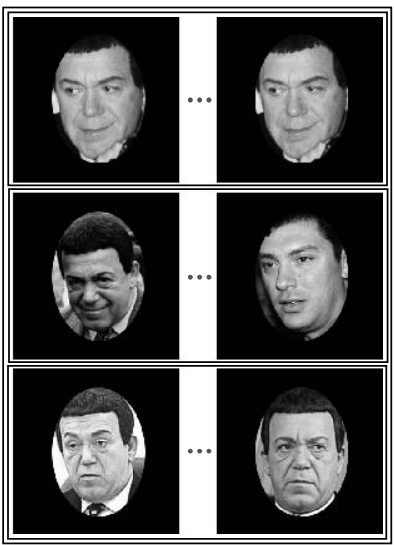

Test:

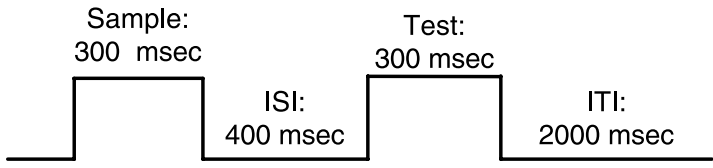

Total trial duration: $3000 \mathrm{msec}$

Figure 2. Design of the face identity matching experiment. (a) In each trial, two faces were presented sequentially and participants performed a 'same/different' identity task. The experiment had a $3 \times 2$ design in which three different trial types were included: same picture, same identity (top row); different picture, different identity (middle row); and different picture, same identity (bottom row). In half of the trials, the pictures were of famous faces and in the other half, they were of unknown faces. The order of the experimental conditions was counterbalanced along the experiment. (b) Schematic of an experimental trial. 
Stimuli were presented at the centre of the screen and a red fixation dot was present throughout the experiment. Each trial lasted $3 \mathrm{sec}$ : the first face stimulus was presented for $300 \mathrm{msec}$ followed by a $400 \mathrm{msec}$ inter-stimulus interval, and then a second face stimulus was presented for $300 \mathrm{msec}$, followed by $2,000 \mathrm{msec}$ inter-trial interval. Participants were instructed to foveate the red fixation dot and to perform a sequential match on identity ('is it the same person?'). The participants were told that it was possible that some of the faces would be familiar to them but that they should perform the task in the same way for each face regardless of familiarity. They completed a short training session to accustom them to the experimental task. Both reaction time and accuracy were recorded using a response box built as a glove to fit to the participants' hand (Psychology Software Tools, Inc. Pittsburgh, PA, USA), and responses were measured from onset of the second face until the end of the trial.

To ensure that any differences obtained in the face identity matching paradigm are not simply attributable to low-level visual differences between the pairs of familiar versus the pairs of unfamiliar faces, we assessed the similarity between the pairs of images in the different experimental conditions using both a physical dissimilarity index and a perceived visual dissimilarity index in a separate behavioural experiment with naive participants who rated the images. The measurement of physical dissimilarity indicated no basic level differences between the two sets of famous and unknown faces. Both the physical and visual dissimilarity indices revealed that pairs of two different images of the same person ('different picture, same identity') were ranked as more similar to each other than pairs of two different individuals ('different picture, different identity'), thus confirming that this condition was the most perceptually challenging condition in this experiment (pairs of identical faces were of course ranked as most similar faces). More details regarding these analysis and the methods employed are described in the Appendix.

This face identity experiment was administered in the fMRI scanner during the course of an experimental scan undertaken for a different purpose. Stimuli were generated using the E-prime software (Psychology Software Tools, Inc. Pittsburgh, PA, USA) and projected via LCD to a screen located in the back of the scanner bore behind the participant's head. The participants viewed the stimuli through a tilted mirror mounted above their eyes on the head coil. Of relevance for the current paper is that the 112 critical images mentioned above were embedded amongst the 560 pictures and the participants performed an identity match on these pictures. This means that, for each participant, we had their overt recognition performance as well as identity match behaviour for the exact same set of faces and it is from these two data sets that we are able to investigate the relationship between covert and overt face processing. CP participants participated in the face identity matching tasks at least several weeks following the completion of the famous face questionnaire (that was used as part of the diagnostic procedure). Control participants completed the questionnaire following the MRI scan during which they performed the face identity matching task. Given these orderings, it is highly unlikely that the completion of the questionnaire could exert any obvious priming effects on the performance of identity matching task.

\section{Calculation of $d^{\prime}$}

In addition to measuring accuracy and RT, we calculated $\mathrm{d}^{\prime}$. To do so, we selected as the 'signal' condition the critical 'different picture, same identity' condition in which the correct response was 'same' and we selected as the 'noise' condition the 'different 
picture, different identity' condition in which the correct response was 'different'. Hits were defined as the number of correct trials in the 'different picture, same identity' condition for a particular level of familiarity divided by the total number of trials in this condition. False alarms were calculated as 1-(number of correct trials in the 'different picture, different identity' condition divided by the total number of trials in this condition). We calculated the hits and false alarms for each familiarity level (famous recognized, famous not recognized, unknown) to obtain a value for $\mathrm{d}^{\prime}$ in each level.

\section{Results}

We first report the results of the $\mathrm{CP}$ participants' performance on the explicit facerecognition task and then we describe the findings from the face identity matching task. Based on the combined analysis of these two tasks, we then examine the dissociation between overt and covert processing.

\section{Famous face questionnaire}

Participants were shown the relevant 112 pictures, half of famous faces and half of unknown faces, and were asked to identify them or provide some contextual information about the faces (Figure 1). Individual percentage correct scores of the $\mathrm{CP}$ participants were compared to two control groups: a. A group of controls who participated in the face identity task during an fMRI scan $(N=12 ; \%$ correct \pm standard error of the mean $=88.69 \pm 4.51$ ) and $\mathrm{b}$. A large control group containing the 12 controls from group ' $a$ ' above and 18 other matched and non-matched controls (total, $N=30 ; \%$ correct \pm standard error of the mean $=83.39 \pm 2.67$ ). All CP participants performed well below the mean of both control groups on the famous faces test. The group differences were assessed by an ANOVA [group (CP, controls) $\times$ response type (correct, don't know, incorrect)]; this analysis revealed a significant interaction of group $\times$ response type and a significant main effect of response type (both effects $p<.0001)$. As can be seen, controls provided significantly more correct responses (planned comparisons: $p<.0001$ ), while CP provided significantly more 'don't know' $(p<.0001)$ and 'incorrect' responses $(p<.02)$. The fact that $\mathrm{CP}$ participants made more errors compared with controls (more 'incorrect' responses) serves as an indication, that at least on the group level, the $\mathrm{CP}$ participants are familiar with popular culture and celebrities and know the names but cannot assign them to the correct face. The statistical difference between each $\mathrm{CP}$ individual and the control group was assessed using the Crawford's modified $t$ test for single case method (Crawford \& Garthwaite, 2002). This calculation was applied to the combined score of correct name and context. Importantly, all $\mathrm{CP}$ participants scored significantly more poorly than control participants (for all CPs except for MT $p<.005$, for MT $p=.05$ ). Note that although CP participant MT's score was relatively high compared with the other CP participants (62.5\%) and so may be considered borderline (but note that his modified $t$ test score is significant $(\phi=.05)$ ), he scored well below four age- and gender-matched controls whose average score was $86.16 \pm 2.28 \%$ thus showing an impairment in famous face recognition $(p<.007$ when MT's score was compared directly with that of his matched controls). It was very rare for either $\mathrm{CP}$ or controls to recognize the unknown faces although occasionally one or two faces (out of the entire 56) would be recognized. 


\section{Face identity matching task}

After establishing the impairment in face recognition in the $\mathrm{CP}$ individuals, all $\mathrm{CP}$ participants and the group of 12 controls participated in the sequential face identity matching task, indicating via button press whether the second face in each pair was of the same person as the first face (Figure 2 and see methods for details). The three trial types included: 'different picture, different identity'; 'different picture, same identity'; and 'different picture, same identity'. It is this third condition that is most critical and in which covert processing, if it exists, might be revealed (the other two conditions might be at ceiling or possibly at floor, respectively). Furthermore, because performance in tasks like this is better for familiar compared with unknown faces even when familiarity is irrelevant (De Haan et al., 1987), we also sought to determine whether and in what way individuals with $\mathrm{CP}$ will be affected by the task-irrelevant factor of face familiarity.

Figure 3 depicts the accuracy and reaction time (RT) for familiar and unknown faces for controls (left panels) and for the CP participants (right panels). A repeated measures ANOVA with familiarity (famous, unknown) and trial type (same picture, same identity; different picture, different identity; different picture, same identity) as within-subject factors and group (control, CP) as a between-subject factor was conducted separately
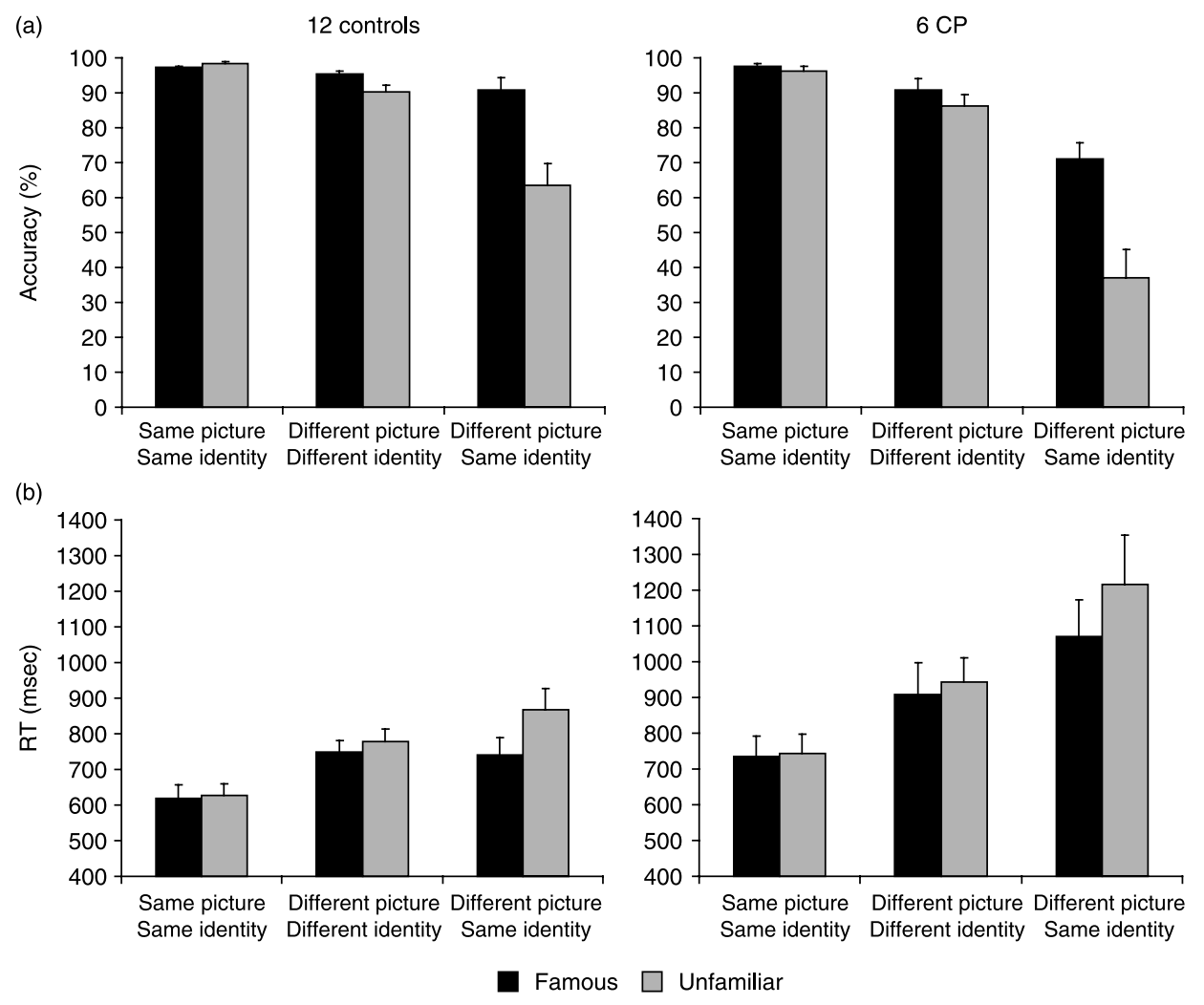

Figure 3. Behavioural performance during the face identity matching task a. Mean of controls' (left panel) and CP's (right panel) accuracy b. Median reaction time for controls and CPs. Overall, both controls and CPs were more accurate and faster in response to the famous faces (black bars) compared with unknown faces (grey bars). The 'different picture, same identity' condition was the most difficult condition, particularly for the unknown faces as evident in both lowest accuracy and slowest RT. 
for accuracy (upper panel) and for median RT (lower panel) of the correct trials. ANOVA for accuracy revealed no three-way interaction $(F(2,32)=0.691)$, but significant twoway interactions of familiarity $\times$ trial type $(F(2,32)=57.24, p<.0001)$, and of group $\times$ trial type $(F(2,32)=5.826, p<.007)$. This latter interaction resulted from the disproportionate drop in performance of the CP group compared with controls on the 'different picture, same identity' trials (planned comparisons: $p<.006$ ). In addition, all main effects were highly significant (familiarity, trial type: $p<.0001$; group: $p<.0002)$. Very similar findings were obtained for the reaction time data: no three-way interaction $(F(2,32)=0.435)$ and significant two-way interactions of familiarity $\times$ trial type $(F(2,32)=35.177, p<.0001)$, and of group $\times$ trial type $(F(2,32)=4.671$, $p<.02$ ). In addition, all main effects were highly significant (familiarity, trial type: $p<.0001$; group: $p<.0002$ ). It is important to note that the slower RT exhibited by the CP individuals compared with controls on the 'different picture, different identity' condition for the unknown faces $(p<.005)$ provides further indication of the perceptual difficulty these individuals have on face discrimination in addition to their impairment in face recognition (see methods and Figure 1b).

These findings indicate that, despite being less accurate and slower, the overall pattern of performance exhibited by $\mathrm{CP}$ individuals was similar to that of controls (importantly, there is no three-way interaction of familiarity $\times$ trial type $\times$ group on either dependent measure). Thus, both groups became less accurate and slower as the task became more difficult but were consistently better at performing the task for the famous faces. However, we do find a two-way interaction of trial type $\times$ group which was due to an overall reduced performance of the $\mathrm{CP}$ group on the critical condition in which two different pictures of the same person were presented. It is the case that, in this critical condition, performance can benefit mostly from face familiarity compared with the other conditions in which the judgment can be resolved on the basis of geometric similarity alone.

\section{Implicit face recognition}

The results presented so far are intriguing since they raise the possibility that, despite the failure to recognize famous faces explicitly, the $\mathrm{CP}$ individuals still process information about these faces in an implicit manner as revealed in superior performance for famous faces over unknown faces even when the two instances of the same face differed in the physical image. However, at this stage, the conclusion that $\mathrm{CP}$ individuals process faces better covertly than overtly is not warranted - for example, the improved performance on famous 'different faces, same identity' could be driven by a subset of the faces that the CP participants just happened to be able to recognize explicitly. Recall that the performance level of the $\mathrm{CP}$ individuals on the famous faces questionnaire was low $(\sim 40 \%)$ but clearly they were still able to recognize some of the faces explicitly.

Thus, in order to make a more specific claim that there is implicit familiarity processing in $\mathrm{CP}$ in the absence of explicit recognition, we needed to ensure that enhanced performance for famous faces was evident not only for those faces recognized overtly but also, more importantly, for those not recognized overtly. To this end, we conducted a subsequent analysis in which we considered simultaneously the performance of the CP from the famous faces questionnaire in relation to the results obtained from the identity-matching experiment. Here, the famous faces trials of the identity-matching experiment were split into trials which included famous faces that each individual CP was able to recognize explicitly as evident in his/her performance of the famous faces questionnaire (we name these trials 'famous recognized') versus trials which included famous faces that were not explicitly recognized ('famous not 
recognized'). The logic behind this analysis is to examine whether famous faces which are not explicitly recognized would still show some privileged processing compared with faces that are completely unknown. Trials where participants recognized only one face but not the other were assigned to the 'famous-recognized' category. Although the focus of this analysis is on the performance of the CP individuals, we also wished to ascertain, as best as possible, how control individuals perform on famous-not recognized faces to provide a rough characterization of what normal implicit performance looks like. However, in order to perform this analysis, one needs to have a reasonable number of trials of famous faces who were not explicitly recognized in the questionnaire and most of the control participants correctly recognized over $90 \%$ of the famous faces included in the explicit questionnaire. As a solution, we selected a subgroup of only 5 controls (out of 12) for whom the performance level was below $90 \%$ and thus their data from the face-matching experiment could be split into trials of famous-recognized faces and famous-not recognized faces. Of note here is that for three of the five controls, we still only had one or two trials in the 'famous not recognized, different picture different identity' condition, and this explains the relatively large variability in this condition (Figure 4 top left graph). Also note that accuracy was calculated as the proportion of trials that were answered correctly from the total number of trials in each category and therefore it need not add up to $100 \%$. The results of this analysis for the subgroup of five controls and six CPs for both accuracy and median RT are presented in Figure 4.

As with the previous analyses, a repeated measures ANOVA was conducted for the split data such that the familiarity factor now had three levels (famous recognized, famous not recognized, unknown). The ANOVA for accuracy revealed no three-way interaction $(F(4,36)=0.62)$ but significant two-way interactions of familiarity level $\times$ trial type $(F(4,36)=6.078, p<.001)$ and of familiarity level $\times$ group $(F(2,18)=3.818, p<.05)$. This latter interaction was due to better performance of the controls than the $\mathrm{CP}$ on the famous-recognized condition than on the unknown face condition (planned comparison, $p<.05$ ). Importantly and most critically, no difference was obtained between $\mathrm{CP}$ and controls in the famous-not recognized condition. In addition, there were significant main effects of trial type $(p<.0003)$ and of familiarity level $(p<.0001)$ but not of group. The RT data generally revealed a similar pattern although here there were no three- or two-way interactions with group and there was a tendency towards a significant main effect of group, indicating that $\mathrm{CP}$ were overall slower compared with controls $(F(1,8)=4.028, p=.08)$.

In light of the significant familiarity level $\times$ trial type interaction in both controls and $\mathrm{CP}$, we went on and conducted planned comparison analyses to examine the effect of familiarity level in our critical experimental condition ('different picture, same identity' across all levels of familiarity). As evident from the graphs in Figure 4, both $\mathrm{CP}$ and controls exhibited a gradual decrease in performance (reduced accuracy, elevated RT) as a function of familiarity and recognition. For the CP group, we found that accuracy did not differ significantly between the famous-recognized and the famous-not recognized condition while both were significantly different $(p<.05)$ from the unknown condition (see Figure 4a, top right panel). In the control group, only the famous-recognized condition was significantly different from the unknown condition $(p<.05)$. As for RT, both CP and controls exhibited similar effects with the famous-recognized and famousnot recognized faces showing a trend towards a significant difference $(p=.055$ and .06 , respectively) and famous-recognized being significantly different from unknown faces $(p<.05)$. 
(a)

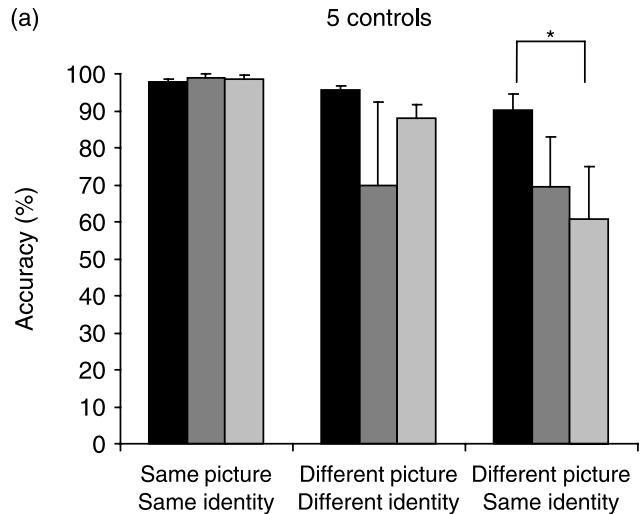

(b)

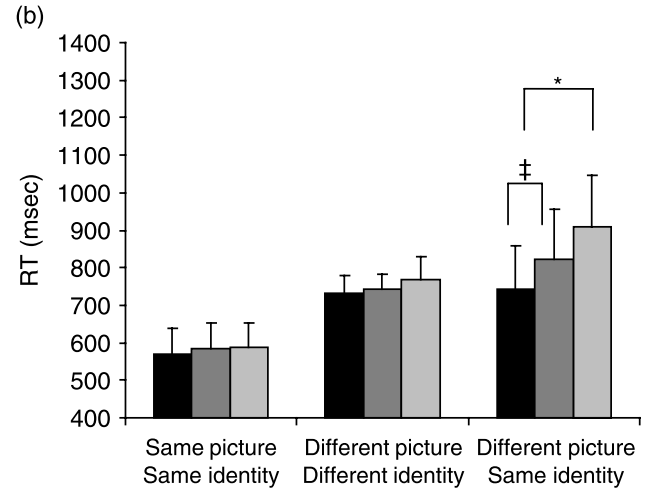

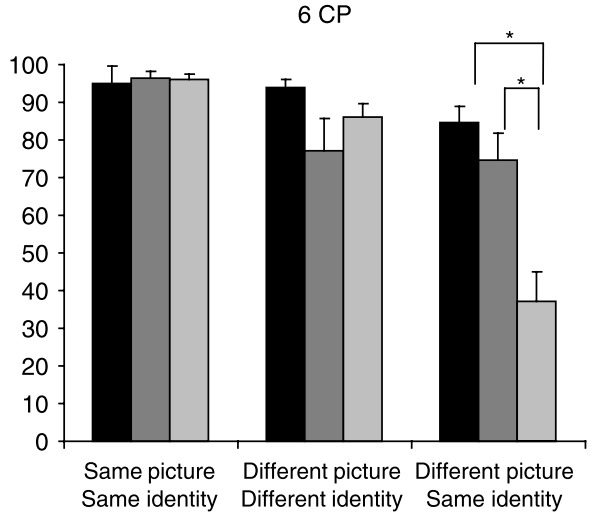

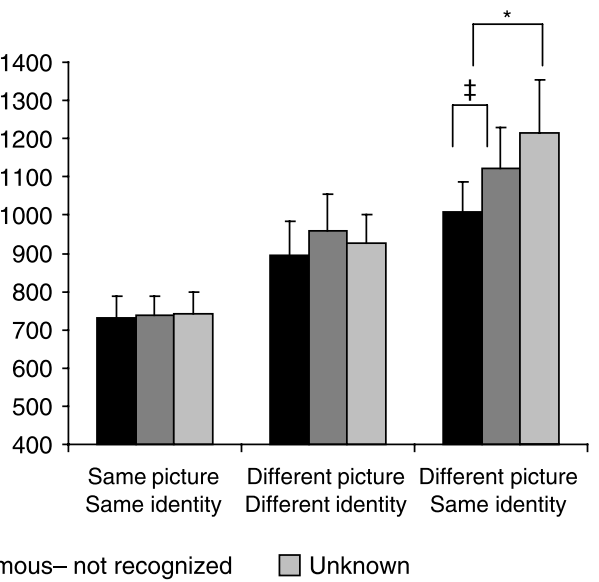

Figure 4. Behavioural performance during the face identity matching task split by explicit recognition measure extracted from the famous faces questionnaire. a. Mean of controls' (left panel) and CP's (right panel) accuracy b. Median reaction time for controls and CPs. As evident, both controls and CPs exhibited an intermediate level of processing in terms of accuracy and RT of famous faces who were not explicitly recognized (dark grey bars) during the 'different picture, same identity' condition. *Significance level of $p<.05$ on planned comparison test. $\neq p$ values which were marginally significant (.06).

In order to test further the differential sensitivity of the CP participants to the three levels of familiarity (famous recognized, famous not recognized, unknown), we calculated the $\mathrm{d}^{\prime}$ or the sensitivity of the participants by comparing their performance on the critical 'different picture, same identity' condition to the 'different picture, different identity' condition. Note that due to the very small number of error trials for three out of five controls in the 'different picture, different identity' condition, we could not conduct a similar analysis for the control group. Means and standard errors of $\mathrm{d}^{\prime}$ values for the three levels of familiarity across the CP group were: $2.34 \pm 0.11$; $1.61 \pm 0.20 ;$ and $0.8 \pm 0.12$. A repeated measures ANOVA revealed that these differences were significant $(F(2,25.94), p<.0003)$ and planned comparison tests revealed a marginally significant difference between the $\mathrm{d}^{\prime}$ for familiar-recognized and familiar not-recognized faces $(p=.06)$, and a significant difference for both of these values from $\mathrm{d}^{\prime}$ for unknown faces $(p<.006)$. These findings provide further corroboration for the differences in performance level found in accuracy and RT. 
Figure 5 shows three graphs summarizing the evidence for implicit processing in $\mathrm{CP}$. The graphs depict the mean of the $\mathrm{CP}$ group as well as data for each individual $\mathrm{CP}$ on all behavioural measures including accuracy, RT, and $\mathrm{d}^{\prime}$ (note that accuracy and RT are redrawn from Figure 4).

As can be seen, in accuracy (Figure 5a), all CP participants are more accurate on famous faces who were not recognized than unknown faces and this same pattern is also true for $\mathrm{d}^{\prime}$ (Figure 5c). In RT, four individuals are faster on famous-not recognized compared with unknown faces; one shows a very similar response to both categories and the other one CP participant shows the reverse response, that is, she is faster on the unknown compared with the famous-not recognized faces. Thus, we show evidence for implicit processing not only at the group level, but also at the individual participant level, such that despite some variability, every CP individual exhibits a privileged response for famous-not recognized faces compared with unknown faces on at least two out of the three behavioural measures we have employed.

Thus, overall, these findings provide evidence that famous faces, which are not explicitly recognized, are processed differently from both famous faces which are explicitly recognized and from unknown faces in that they elicit an intermediate level of performance compared with the other two classes. Hence, although individuals with $\mathrm{CP}$ are slower and less accurate compared with controls, they still exhibit evidence for implicit familiarity processing. The implications of these findings to models of $\mathrm{CP}$ are discussed below.

\section{Discussion}

This study provides clear evidence for the existence of implicit familiarity processing in a relatively large group of individuals with $\mathrm{CP}$. We first documented their difficulty in explicit face recognition using a famous face questionnaire and then examined their implicit face-processing abilities in a face identity matching task. To evaluate specifically the ability to make decisions on famous faces whom they could not explicitly recognize, we performed a combined analysis taking into account simultaneously the performance on the explicit and implicit tasks. Despite being slower and less accurate than the controls, the $\mathrm{CP}$ individuals exhibited a behavioural pattern very similar to that of the controls. Both groups responded faster and more accurately for pairs of famous compared with unfamiliar faces and both performed better on trials where the two images of a face were identical compared with trials on which the two images of the same individual were different. But of greatest interest, the combined analysis revealed that, for both groups, the performance level for famous faces that were not explicitly recognized was set at an intermediate level between famous faces that were explicitly recognized and faces that were completely unknown, and this rank ordering was only evident in the critical experimental condition in which participants had to match two different pictures of the same person. Of note is that this critical condition is the most challenging condition compared with the two other experimental conditions ('same picture, same identity'; 'different picture, different identity'). This was evident from two independent measures which examined the physical and perceptual dissimilarity of the faces in each condition; these measures revealed that two different images of the same person are ranked as being more similar to each other than two images of two different people (see Appendix for more details). Thus, it is in this condition where any representation about identity and familiarity can maximally enhance performance - in the two other conditions, identity decision can be resolved solely on the basis of the geometrical or featural properties of the images and can also be subject to floor/ceiling effects. 

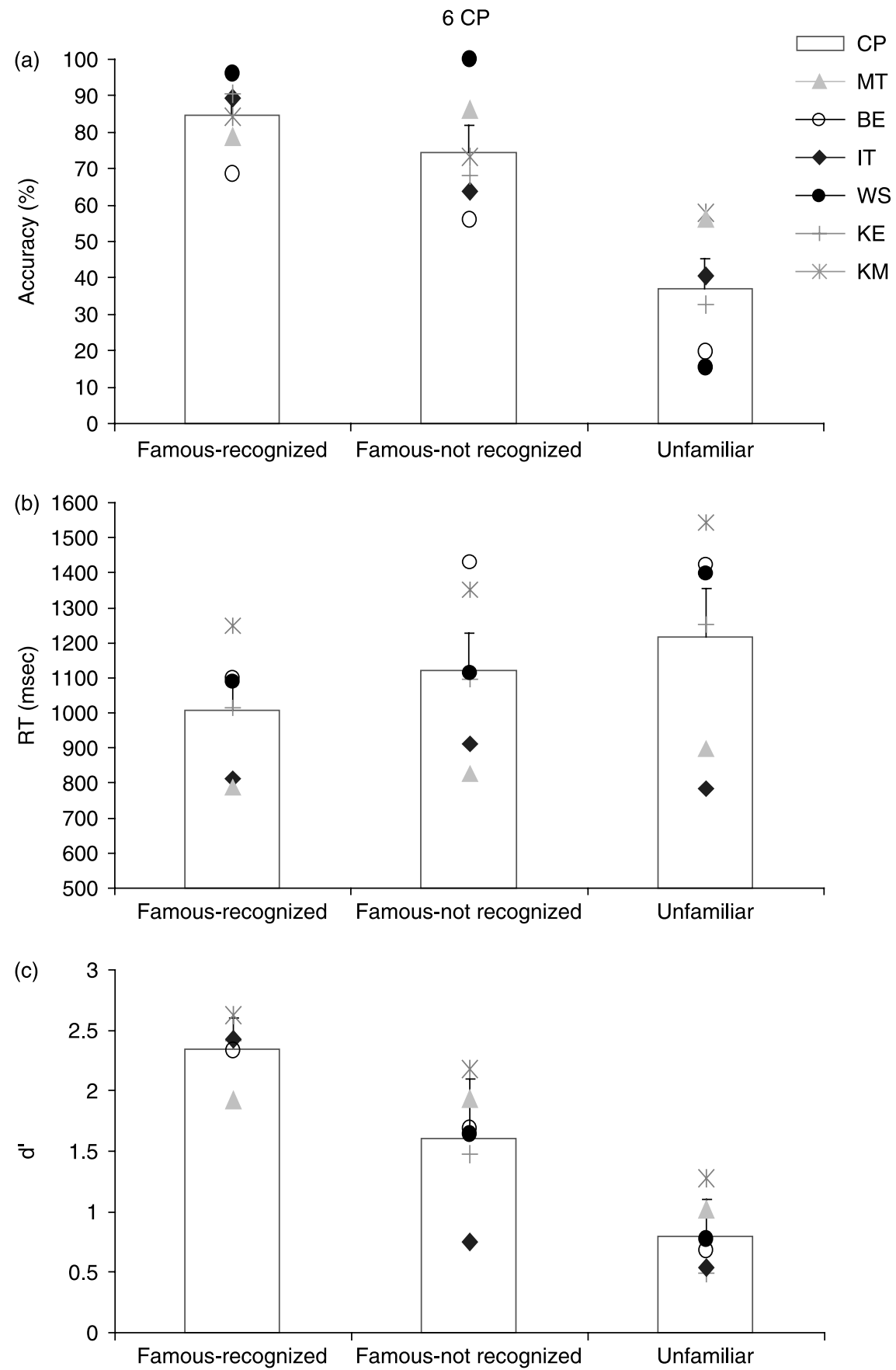

Figure 5. Summary of evidence for implicit processing in CP. Mean (grey bars) and individual data of CP participants (different symbols) showing evidence for implicit processing on three different behavioural measures: (a) accuracy calculated for the critical 'different picture, same identity' condition for each level of familiarity (famous recognized, famous not recognized, unknown). (b) median RT calculated as in a. (c) d' calculated by comparing participant's performance on the 'different picture, same identity' condition to the 'different picture, different identity' condition for each level of familiarity. 


\section{Implicit processing in other cases of CP}

To date, as reviewed in the Introduction, only a few studies have examined implicit processing in $\mathrm{CP}$ and the available evidence is somewhat mixed. Two studies report no evidence for implicit processing in CP (Bentin et al., 1999; De Haan \& Campbell, 1991), although Jones and Tranel (2001) do report increased SCR amplitude for famous compared with unfamiliar faces in a child with developmental/congenital prosopagnosia. It is not clear that the mechanisms underlying increased SCR are the same as those underlying successful implicit recognition as evident in more conventional behavioural studies and so it may be difficult to compare these mixed results (for a discussion of this issue in AP, see Schweinberger \& Burton, 2003).

We note however, that a very recent study (Bentin et al., 2007) uncovered some evidence suggestive of implicit processing in a behavioural task in which their $\mathrm{CP}$ participant, KW, had to match either the identity or the expressions of unfamiliar faces. Under these conditions, KW exhibited better expression matching for faces that shared the same identity than for those that did not share identity. This beneficial performance for shared identity images was taken as indirect evidence for covert processing. The exact interpretation of this finding is not completely clear, however. In the first instance, because this experiment was conducted with unfamiliar faces, the matching could not be done using any pre-existing face representation or knowledge about identity per se and so the mechanism of implicit priming may be different from that which supports facilitation by familiarity. Indeed, there is ongoing controversy as to whether or not famous faces are processed in the same way as unknown faces (Hancock, Bruce, \& Burton, 2000), and so it is difficult to predict whether the facilitated expression processing by shared identity would also be replicated with famous faces. Moreover, expressions vary substantially between people so it is not entirely surprising that $\mathrm{KW}$ is better at expression matching produced by the same person than those produced by different individuals.

One possible explanation for the enhanced performance reported by Bentin et al. $(2007,1999)$ is that if the representation of expression is preserved in KW (as it is in many other cases of CP, see Duchaine et al., 2007; Humphreys et al., 2006), then deriving the expression or dynamic aspects of a face (which may be shared or even invariant across different pictures of the same individual) may prime some aspects of its identity and thus indirect activation of identity can be used to enhance expression matching in same versus different trials. Indeed, there is recent support for the idea that identity and facial expression are not computed independently and so accessing one aspect of a face may assist activation of other aspects of the face too (Ganel, Valyear, Goshen-Gottstein, \& Goodale, 2005). It is important to note that participant $\mathrm{KW}$ failed to show evidence for implicit processing when more conventional tasks for implicit recognition were applied.

It is clear that further studies of implicit processing in CP are warranted in order to understand how general the phenomenon is and under what circumstances and paradigms it can be elicited (for an analogous discussion in AP, see Barton et al., 2004). Nevertheless, the present findings, which provide the first systematic evaluation of implicit processing of both famous and unknown faces in a relatively large group of participants, suggests that this effect may be quite robust.

\section{Mechanisms supporting implicit processing}

In contrast to $\mathrm{CP}$ in which the presence of implicit effects is not yet established fully, in AP there is considerable empirical evidence in support of the presence of implicit face processing in many, although not all individuals, and this is true even in cases with 
severe impairments in explicit face recognition (Barton et al., 2004; Bruyer, 1991; Farah et al., 1993). Several different theoretical accounts have been proposed to explain this finding, and we examine whether and to what extent these apply to $\mathrm{CP}$ too. We proceed cautiously in doing so as, even in AP, there is not a single model for implicit processing which is unanimously accepted and, at this stage, the empirical evidence does not allow us to unequivocally distinguish between contrasting views (see Bobes et al., 2003; Sperber \& Spinnler, 2003). We also note that despite the general similarity of symptoms in $\mathrm{CP}$ and $\mathrm{AP}$, the analogy between these two disorders is not entirely straightforward and their core characteristics do not line up perfectly (for differences in facial expression representation, see Humphreys et al., 2007 and see Behrmann, Avidan, \& Humphreys, 2008 for a broader discussion of this issue). Also, like AP, CP is almost certainly a heterogeneous disorder (Behrmann et al., 2005; Bentin et al., 2007; Duchaine \& Nakayama, 2004; Le Grand et al., 2006), and different individuals exhibit variations in the nature and severity of the symptoms. Having laid out the caveats up front, we now explore the applicability of the models of implicit processing in AP to CP.

Perhaps the most interesting difference between AP and CP and the crux of the issue addressed here is whether these AP models have any applicability to $\mathrm{CP}$ given that, inherent in them is the assumption of the prior existence of face representations. This assumption is entirely reasonable given that AP individuals are, by definition, premorbidly normal with normal acquisition of face representations. Whether these pre-existing representations are necessary for implicit processing is important to understand and CP individuals who, by definition, do not have a fully developed 'premorbid' set of face representations provide an excellent opportunity to explore this issue.

Several explanations have been offered to account for the discrepancy between intact covert processing and impaired overt processing in AP. Bauer (1984), for example, suggested that there are two face-processing streams such that overt recognition is accomplished by the ventral visual pathway, while covert recognition is mediated by a dorsal visual limbic pathway. In AP the overt, ventral system is damaged and so the patients are left with only the output of the dorsal system subserving covert processing. Although this model may account for covert processing in AP when affective responses are implicated (such as in cases of facial expression judgment or in some skin conductance tasks), many implicit tasks do not involve any affective response related to the face and, rather, involve knowledge about occupations or names of individual. It is not clear, then, how these implicit tasks could be resolved according to this account. A rather different conceptual model, offered by De Haan and colleagues (1992), is that, in AP, there is a disconnection of an intact face-recognition system enabling covert recognition from a higher cognitive system enabling conscious awareness.

In contrast with these accounts, a further alternative class of models argue that both covert and overt processing may be achieved by a single face-processing system. Burton and colleagues (1991) have proposed a model which, like De Haan and colleagues (1992), is also essentially a disconnection model but, in contrast with that model, the disconnection occurs between subunits of the face-recognition system such that information from the intact visual recognition units (face-recognition units - FRU, in the notation of the Bruce and Young model (Bruce \& Young, 1986)) cannot access the rest of the face-recognition system and particularly the modality-independent person representations (PIN - personal identity units) which are essential for explicit recognition. A final account proposed by Farah and colleagues (1993) strongly favours a view in which overt and covert recognition is accomplished by a single neural system, rather than by one subsystem for perceptual aspects of face processing and by one permitting conscious awareness. Also, in contrast to 
other models, they acknowledge that face representation in AP is not intact but rather is degraded or incomplete. Nevertheless, they claim that the partial face representation may still allow successful covert recognition but is not enough to support overt recognition, which is more taxing and demanding.

We now examine with which model/s the empirical evidence gathered so far in $\mathrm{CP}$ fit best. Given that many individuals with CP exhibit difficulties in perceptual tasks involving unfamiliar faces or even other visual tasks involving non-face objects (Behrmann et al., 2005; Bentin et al., 2007; Duchaine et al., 2007), the assumption of intact perception in both the De Haan and Burton's models rules them out. We also note that perceptual difficulties are manifested in the present study in that the CP individuals perform more poorly than the controls on matching unfamiliar faces (see Methods) and on the experimental face identity matching task which is essentially a perceptual task particularly for the unknown faces (Figure 3). In the Farah model, there is clear recognition that there is impaired face representation in AP and the core notion of this account is that, in AP, the degraded face representations suffice for intact covert but not overt recognition. The same argument is easily applicable in the case of $\mathrm{CP}$ - the partial representations that the $\mathrm{CP}$ individuals may have developed over time may suffice for covert but not overt face processing given the differential sensitivity of these two tasks. It is important to note that the CP individuals tested here do have some, perhaps incomplete or partial, set of face representation as they do not score zero on the famous faces questionnaire or on the face identity matching task. Thus, they have managed to acquire some face representations, even if not normal or fully intact, and these can be activated under certain circumstances. The key finding is that, whatever the nature of the underlying representations, and their exact nature remains to be determined, they may be inadequate to support overt recognition but, as in AP, may be sufficient for covert judgments.

Thus, on this account, implicit processing is possible even if prior normal representations do not exist, in contrast with the view proposed by Schweinberger and Burton (2003) and also implied by Farah and colleagues (1993). Consistent with this is that some patients with AP exhibit covert processing not only for faces they knew before their injury and for whom they likely had prior normal representation, but also for faces of people they met only following the injury and were not explicitly recognized (i.e. anterograde prosopagnosia) (De Haan et al., 1987; Tranel \& Damasio, 1985). These examples imply that new face representations may still be created (perhaps partially or with incomplete precision) even when the face-recognition system is damaged. These novel representations may then be sufficient to enable covert recognition but not overt recognition. A similar process may explain the evidence for implicit processing in CP: multiple exposures to famous faces throughout their life allows them to build some (even if partial or degraded) representation for these faces. In light of these findings, it will be of great interest to explore whether the neural network model employed by Farah and colleagues (1993) with AP and which assumes an initial intact face representation can be modified to account for the findings obtained with $\mathrm{CP}$ in whom such representations were never completely normal and for the presence of implicit processing in AP for newly acquired face representations.

Although our data might fit well with the approach espoused by Farah and colleagues, they are also compatible with some aspects of the disconnection approach of Burton et al. (1991). We note that the different models are not necessarily mutually exclusive (for a relevant discussion regarding the possible integration of different models, see Farah, 1992). In a recent in-depth analysis of CP (Thomas et al., 2006), we employed diffusion tensor tractography to examine the structural integrity of the multiple regions of the brain 
known to be involved in face processing (for an overview of this network, see Gobbini \& Haxby, 2007). The major result from this investigation is the apparent reduction in white matter integrity in $\mathrm{CP}$ individuals in the tracts that project through the fusiform gyrus to more anterior temporal regions as well as to frontal cortex. These results are also consistent with evidence for reduced volume in the anterior fusiform gyrus in these same individuals (Behrmann et al., 2007). The findings are compatible with the view that, within the single face network, there may be a disconnection between more posterior regions and more anterior temporal and frontal regions, which are part of the extended face-processing system (Gobbini \& Haxby, 2007; Haxby et al., 2001). We note that these extended regions might serve the role of the higher-order areas which are necessarily engaged for successful overt recognition in the model of Burton et al. There is also empirical evidence suggesting that such regions are involved in subordinate level recognition or individual recognition (Puce, Allison, \& McCarthy, 1999).

Thus, at this stage of our investigations, we know that CP individuals perform poorly at face perception tasks as well as at recognition. The disorder might arise from an initial disruption in perceptual processing, perhaps mediated by more posterior cortical regions, which is then accompanied by disrupted propagation of this information into other more anterior regions. It is also possible that a more posterior impairment which precludes the acquisition of detailed perceptual representations leads to the propagation (perhaps both feedforward and feedback) of only weak input into anterior regions and this, during the course of development, results in the formation of only weak connections between these subregions, as evident by Diffusion Tensor Imaging. An alternative possibility is that the entire integrated network is necessary for the computation of representations for perceptual discrimination and for recognition, and that the circuitry from more posterior to more extended regions might play a role in the computation of these foundational representations, for example, in deriving the higherorder statistics from the input. Partial computation may be possible and this could support the covert but not overt performance in CP.

To conclude, we believe that the present findings are very important for our understanding of the underlying mechanism of CP. As in the case of AP, further studies are clearly warranted in order to determine the exact neural mechanism allowing covert recognition, but this study, which provides strong evidence for the existence of such form of processing, is a stepping stone in that direction. Finally, we believe that the existence of implicit processing in $\mathrm{CP}$ may be of great relevance to intervention studies in these individuals as it implies that some knowledge about familiar people does exist in their face-recognition system. It may be possible then that, with the appropriate training, such knowledge may become more accessible for these individuals.

\section{Acknowledgements}

We thank Grace Lee Leonard for her substantial help with stimulus preparation and testing, and Michal Tanzer for help with data analysis. We also thank Cibu Thomas for his help in testing participants KE and WS and for data analysis. This work was supported by a NIMH 54246 grant to MB.

\section{References}

Avidan, G., Hasson, U., Malach, R., \& Behrmann, M. (2005). Detailed exploration of face-related processing in congenital prosopagnosia: 2. Functional neuroimaging findings. Journal of Cognitive Neuroscience, 17(7), 1150-1167. 
Avidan, G., Thomas, C. P., \& Behrmann, M. (2007). An integrative approach towards understanding the psychological and neural basis of congenital prosopagnosia. In I. Harris \& M. Jenkin (Eds.), Cortical mechanisms of vision, Forthcoming.

Barton, J. J. (2003). Disorders of face perception and recognition. Neurologic Clinics, 21(2), 521-548.

Barton, J. J., Cherkasova, M. V., \& Hefter, R. (2004). The covert priming effect of faces in prosopagnosia. Neurology, 63(11), 2062-2068.

Barton, J. J., Cherkasova, M. V., Press, D. Z., Intriligator, J. M., \& O'Connor, M. (2003). Developmental prosopagnosia: A study of three patients. Brain and Cognition, 51(1), 12-30.

Bauer, R. M. (1984). Autonomic recognition of names and faces in prosopagnosia: A neuropsychological application of the Guilty Knowledge Test. Neuropsychologia, 22(4), 457-469.

Behrmann, M., \& Avidan, G. (2005). Congenital prosopagnosia: Face-blind from birth. Trends in Cognitive Sciences, 9(4), 180-187.

Behrmann, M., Avidan, G., Gao, F., \& Black, S. (2007). Structural imaging reveals anatomical alterations in inferotemporal cortex in congenital prosopagnosia. Cerebral Cortex, 17(10), 2354-2363.

Behrmann, M., Avidan, G., \& Humphreys, K. (2008). Congenital and Acquired Prosopagnosia. In D. Bub, I. Gauthier \& M. Tarr (Eds.), Perceptual Expertise: Bridging Brain and Behavior. Oxford University Press.

Behrmann, M., Avidan, G., Marotta, J. J., \& Kimchi, R. (2005). Detailed exploration of face-related processing in congenital prosopagnosia: 1. Behavioral findings. Journal of Cognitive Neuroscience, 17(7), 1130-1149.

Bentin, S., Degutis, J. M., D'Esposito, M., \& Robertson, L. C. (2007). Too many trees to see the forest: Performance, event-related potential, and functional magnetic resonance imaging manifestations of integrative congenital prosopagnosia. Journal of Cognitive Neuroscience, 19(1), 132-146.

Bentin, S., Deouell, L. Y., \& Soroker, N. (1999). Selective visual streaming in face recognition: Evidence from developmental prosopagnosia. Neuroreport, 10(4), 823-827.

Bobes, M. A., Lopera, F, Garcia, M., Diaz-Comas, L., Galan, L., \& Valdes-Sosa, M. (2003). Covert matching of unfamiliar faces in a case of prosopagnosia: An ERP study. Cortex, 39(1), 41-56.

Bodamer, J. (1947). Die prosop-Agnosie. Archiv für Psychiatrie und Nervenkrankheiten, 179, 6-53.

Bouvier, S. E., \& Engel, S. A. (2006). Behavioral deficits and cortical damage loci in cerebral achromatopsia. Cerebral Cortex, 16(2), 183-191.

Bruce, V., \& Young, A. (1986). Understanding face recognition. British Journal of Psychology, 77(Pt 3), 305-327.

Bruyer, R. (1991). Covert face recognition in prosopagnosia: A review. Brain and Cognition, 15(2), 223-235.

Burton, A. M., Young, A. W., Bruce, V., Johnston, R. A., \& Ellis, A. W. (1991). Understanding covert recognition. Cognition, 39(2), 129-166.

Crawford, J. R., \& Garthwaite, P. H. (2002). Investigation of the single case in neuropsychology: Confidence limits on the abnormality of test scores and test score differences. Neuropsychologia, 40(8), 1196-1208.

Damasio, A. R., Damasio, H., \& Van Hoesen, G. W. (1982). Prosopagnosia: Anatomic basis and behavioral mechanisms. Neurology, 32(4), 331-341.

De Haan, E. H., Bauer, R. M., \& Greve, K. W. (1992). Behavioural and physiological evidence for covert face recognition in a prosopagnosic patient. Cortex, 28(1), 77-95.

De Haan, E. H., \& Campbell, R. (1991). A fifteen year follow-up of a case of developmental prosopagnosia. Cortex, 27(4), 489-509.

De Haan, E. H., Young, A., \& Newcombe, F. (1987). Face recognition without awareness. Cognitive Neuropsychology, 4(4), 385-415.

Duchaine, B., Germine, L., \& Nakayama, K. (2007). Family resemblance: Ten family members with prosopagnosia and within-class object agnosia. Cognitive Neuropsychology, 24(4), 419-430. 
Duchaine, B. C., \& Nakayama, K. (2004). Developmental prosopagnosia and the Benton Facial Recognition Test. Neurology, 62(7), 1219-1220.

Farah, J. M. (1992). Visual perception and visual awareness after brain damage: A tutorial overview. In C. Umilta \& M. Moscovitch (Eds.), Attention and Performance $X V$ (pp. 35-76). Cambridge, MA: The MIT Press.

Farah, M. J., O'Reilly, R. C., \& Vecera, S. P. (1993). Dissociated overt and covert recognition as an emergent property of a lesioned neural network. Psychological Review, 100(4), 571-588.

Ganel, T., Valyear, K. F., Goshen-Gottstein, Y., \& Goodale, M. A. (2005). The involvement of the 'fusiform face area' in processing facial expression. Neuropsychologia, 43(11), 1645-1654.

Gobbini, M. I., \& Haxby, J. V. (2007). Neural systems for recognition of familiar faces. Neuropsychologia, 45(1), 32-41.

Grill-Spector, K., Kushnir, T., Edelman, S., Avidan, G., Itzchak, Y., \& Malach, R. (1999). Differential processing of objects under various viewing conditions in the human lateral occipital complex. Neuron, 24(1), 187-203.

Grueter, M., Grueter, T., Bell, V., Horst, J., Laskowski, W., Sperling, K., et al. (2007). Hereditary prosopagnosia: The first case series. Cortex, 43(6), 734-749.

Hadjikhani, N., \& De Gelder, B. (2002). Neural basis of prosopagnosia: An fMRI study. Human Brain Mapping, 16(3), 176-182.

Hancock, P. J., Bruce, V. V., \& Burton, A. M. (2000). Recognition of unfamiliar faces. Trends in Cognitive Sciences, 4(9), 330-337.

Harris, A. M., Duchaine, B. C., \& Nakayama, K. (2005). Normal and abnormal face selectivity of the M170 response in developmental prosopagnosics. Neuropsychologia, 43(14), 2125-2136.

Hasson, U., Avidan, G., Deouell, L. Y., Bentin, S., \& Malach, R. (2003). Face-selective activation in a congenital prosopagnosic subject. Journal of Cognitive Neuroscience, 15(3), 419-431.

Haxby, J. V., Gobbini, M. I., Furey, M. L., Ishai, A., Schouten, J. L., \& Pietrini, P. (2001). Distributed and overlapping representations of faces and objects in ventral temporal cortex. Science, 293(5539), 2425-2430.

Humphreys, K., Avidan, G., \& Behrmann, M. (2007). A detailed investigation of facial expression processing in congenital prosopagnosia as compared to acquired prosopagnosia. Experimental Brain Research, 176(2), 356-373.

Jones, R. D., \& Tranel, D. (2001). Severe developmental prosopagnosia in a child with superior intellect. Journal of Clinical and Experimental Neuropsychology, 23(3), 265-273.

Kanwisher, N., McDermott, J., \& Chun, M. M. (1997). The fusiform face area: A module in human extrastriate cortex specialized for face perception. Journal of Neuroscience, 17(11), 4302-4311.

Kress, T., \& Daum, I. (2003). Event-related potentials reflect impaired face recognition in patients with congenital prosopagnosia. Neuroscience Letters, 352(2), 133-136.

Le Grand, R., Cooper, P. A., Mondloch, C. J., Lewis, T. L., Sagiv, N., de Gelder, B., et al. (2006). What aspects of face processing are impaired in developmental prosopagnosia? Brain and Cognition, 61(2), 139-158.

Minnebusch, D. A., Suchan, B., Ramon, M., \& Daum, I. (2007). Event-related potentials reflect heterogeneity of developmental prosopagnosia. European Journal of Neuroscience, 25(7), 2234-2247.

Puce, A., Allison, T., \& McCarthy, G. (1999). Electrophysiological studies of human face perception. III: Effects of top-down processing on face-specific potentials. Cerebral Cortex, 9(5), 445-458.

Renault, B., Signoret, J. L., Debruille, B., Breton, F., \& Bolgert, F. (1989). Brain potentials reveal covert facial recognition in prosopagnosia. Neuropsychologia, 27(7), 905-912.

Rizzo, M., Hurtig, R., \& Damasio, A. R. (1987). The role of scanpaths in facial recognition and learning. Annals of Neurology, 22(1), 41-45.

Schweinberger, S. R., \& Burton, A. M. (2003). Covert recognition and the neural system for face processing. Cortex, 39(1), 9-30. 
Sperber, S., \& Spinnler, H. (2003). Covert person recognition: Its fadeout in a case of temporal lobe degeneration. Cortex, 39(1), 57-67.

Thomas, C. P., Avidan, G., Jung, K. J., \& Behrmann, M. (2006). Disruption in structural connectivity in ventral visual cortex in congenital prosopagnosia. 6th Annual Vision Sciences Society Meeting, Florida.

Tranel, D., \& Damasio, A. R. (1985). Knowledge without awareness: An autonomic index of facial recognition by prosopagnosics. Science, 228(4706), 1453-1454.

Young, A. W., Hellawell, D., \& De Haan, E. H. (1988). Cross-domain semantic priming in normal subjects and a prosopagnosic patient. Quarterly Journal of Experimental Psychology. A, 40(3), 561-580.

Young, A. W., McWeeny, K. H., Hay, D. C., \& Ellis, A. W. (1986). Matching familiar and unfamiliar faces on identity and expression. Psychological Research, 48(2), 63-68.

Received 3 July 2007; revised version received 6 November 2007

\section{Appendix}

\section{Assessing the level of similarity between stimuli}

To ensure that the behavioural results obtained using the face identity matching paradigm cannot simply be accounted for by low-level visual differences between the pairs of famous and unknown faces, we assessed the similarity between the pairs of images in the different experimental conditions using both a physical dissimilarity index and a perceived visual similarity index in a separate behavioural experiment with naïve participants who rated the images.

\section{Physical dissimilarity index}

To estimate the physical similarity between pairs of images from each condition in the famous and unknown sets, the point-wise Euclidian distance between pairs of images was analysed using a method previously described (Grill-Spector et al., 1999). Formally, the dissimilarity index is defined as follows:

$$
d_{j k}=\frac{1}{n} \sqrt{\sum_{x=1}^{n}\left[I_{j}(x)-I_{k}(x)\right]^{2}} \quad[j, k=1, \ldots, p]
$$

Such that $n$ indicates the number of pixels within an image, $I_{j}(x)$ indicates the grey level value of the pixel in location $x$ in the image $I_{j}$, and $p$ is the number of all images belonging to a specific condition. The mean point-wise distance $(d)$ over all images in a condition, which is termed the physical dissimilarity index, was calculated as $d=E\left(d_{j k}\right)$. Thus, the analysis yielded a dissimilarity index (the larger the index, the greater the dissimilarity is) for each pair of stimuli and then this index was summed overall pairs of stimuli within a condition. The physical dissimilarity of pairs of identical stimuli was, of course, zero and, therefore, these conditions were not included in further statistical analysis. A familiarity $\times$ condition ANOVA revealed a significant main effect of condition $(p<.0001)$ : unsurprisingly, pairs of faces in the 'different picture, different identity' condition were more dissimilar than pairs in the 'different picture, same identity'. Critically, there was no main effect of familiarity $(p=.2)$ nor an interaction between familiarity and condition $(p=.6)$, confirming that familiar and unfamiliar pairs of faces within each condition were physically equivalently similar to each other. 


\section{Perceived visual dissimilarity index}

The purpose of this analysis was to estimate the perceived visual similarity between pairs of faces in each experimental condition, based on observers' judgments. The experimental design was identical to that used for the face identity matching experiment except that the participants were required to rank the visual similarity between the two faces in each trial rather than make a same/different identity judgment between them. Ranking ranged between 1 and 4 , with ' 1 ' indicating very similar faces and ' 4 ' indicating very different faces. The experiment was conducted on a PC and was executed using the E-prime software (Psychology Software Tools, Inc. Pittsburgh, PA, USA). Participants responded by pressing one of four keys marked on the keyboard. Participants sat at a viewing distance of approximately $60 \mathrm{~cm}$ from the computer screen. Ten participants (five females), aged 18-22, participated in this experiment, none of whom participated in any of the tasks included in the main paper.

Interestingly, the results obtained in this task suggested that participants' perceptions are influenced by the familiarity of the face. A familiarity $\times$ condition ANOVA revealed a significant interaction between familiarity $\times$ condition factors $(p<.0001)$ as well as significant main effects of both factors $(p<.0002)$. Similar to the ranking obtained in the physical dissimilarity index, participants ranked the two different images of the same person (of both famous and unknown faces) as being more similar to each other than two images of two different people $(p<.00001)$ and were also slower when ranking the 'same person, different identity' pairs $(p<.002)$. Interestingly, however, and in contrast with the physical dissimilarity index, participants perceived the famous 'different picture, same identity' pairs as more similar to each other than the unknown pairs of the same condition $(p<.05$, Tukey's post hoc test), as reflected both in their rankings and their RTs (slower for unfamiliar pairs) $(p<.05)$. These perceptual results are interesting and are consistent with previous findings reported in the literature (De Haan et al., 1987). Note that face familiarity was not relevant to the behavioural task during the face identity matching task or the ranking task and yet familiarity significantly influences perception. These findings provide further evidence for implicit familiarity processing in normal individuals on an additional task which is different from the one we used in the main experiment reported in this paper. 